\title{
Analysis and Modeling of the Quasi-Z-Source-Inverter
}

\author{
Andreas Liske, Gerhard Clos and Michael Braun \\ Karlsruhe Institute of Technology (KIT) \\ Elektrotechnisches Institut (ETI) - Electrical Drives and Power Electronics \\ Kaiserstr. 12, 76131 Karlsruhe, Germany \\ andreas.liske@kit.edu,www.eti.kit.edu
}

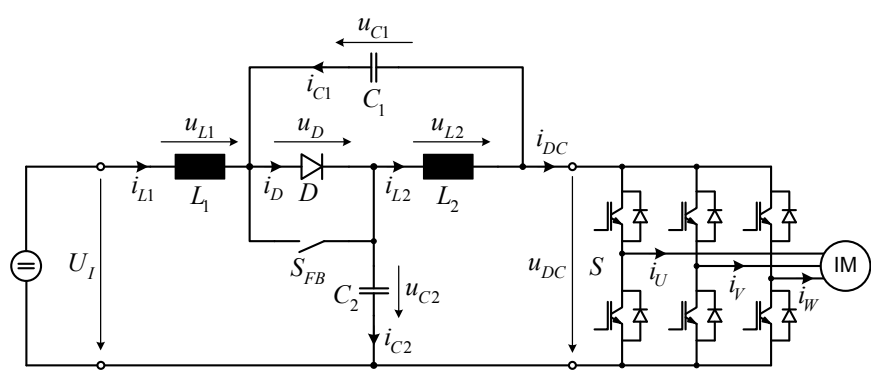

Figure 1. Quasi-Z-Source-Inverter with feedback switch $S_{F B}$

\begin{abstract}
This paper presents a dynamic average model of the Quasi-Z-Source Inverter (QZSI), based on the short term average values over one PWM-period, and the analysis of the possible switching states that can occur in the QZSI. The model is considerably descriptive since the modeling has been performed by analyzing the physical and functional relations with only little mathematical complexity. Another advantage of the proposed model is that the load current only acts as a disturbance input, and is not directly linked with the model structure itself. This allows a separate examination of either command input or disturbance response and makes the model independent of the given load. The model has been tested successfully in conjunction with an adequate cascade control loop design and a QZSIprototype in hardware.
\end{abstract}

\section{INTRODUCTION}

The QZSI is a variation of the Z-Source-Inverter (ZSI) ([1], [2], [3]). It combines the function of a conventional boost-converter with the inverter-function of a full bridge inverter in one circuit (fig. 1). Several papers have already been published describing the modeling of the ZSI with the known techniques of state-space-averaging ([4],[5],[6],[7]). In this paper, the approach to gain a dynamic average model of the Quasi-ZSI is to analyze the possible switching states and the functional relations in the circuit and how they can be used to control the QZSI. In chapter II of this paper, a brief description of the basic function and the steady state behavior of the QZSI is given and the possible circuit switching states that can occur in the QZSI are described. It can be shown that for $L_{1}=L_{2}$ and $C_{1}=C_{2}$ the time values of the inductor currents and the differential capacitor voltages are equal in every possible switching state. Based on this analysis, the dynamic average model of the QZSI is devel- oped in chapter III. The model was taken as a basis for an adequate control loop design and tested in a QZSI-hardwareprototype that was designed for $10 \mathrm{~kW}$ at $f_{P W M}=10 \mathrm{kHz}$, $U_{I}=200 . .400 \mathrm{~V}$ and $\bar{u}_{D C}=400 . .600 \mathrm{~V}$. The passive components' values were $L_{1}=L_{2}=1,8 \mathrm{mH}$ and $C_{1}=C_{2}=100 \mu \mathrm{F}$. The functional test was performed at low voltage with $U_{I}=40 \mathrm{~V}$ and $\bar{u}_{D C, w}=u_{C 2, w}=50 \mathrm{~V}$ with $f_{P W M}=10 \mathrm{kHz}$. The test results prove the theory completely. Chapter IV is a summary of the experimental results.

\section{Possible Switching States of the QZSI}

The boost-function of the QZSI is achieved by applying a short-circuit (Shootthrough) to the DC-Bus periodically. During this shootthrough, the inductor currents are rising and energy is stored in the inductors. When the shootthrough is terminated, this energy is being transferred into the capacitors and the load. The capacitor voltages are rising and the inductor currents are sinking again. The steady-state operational behavior of the QZSI in boost operating mode is shown in fig. 2.

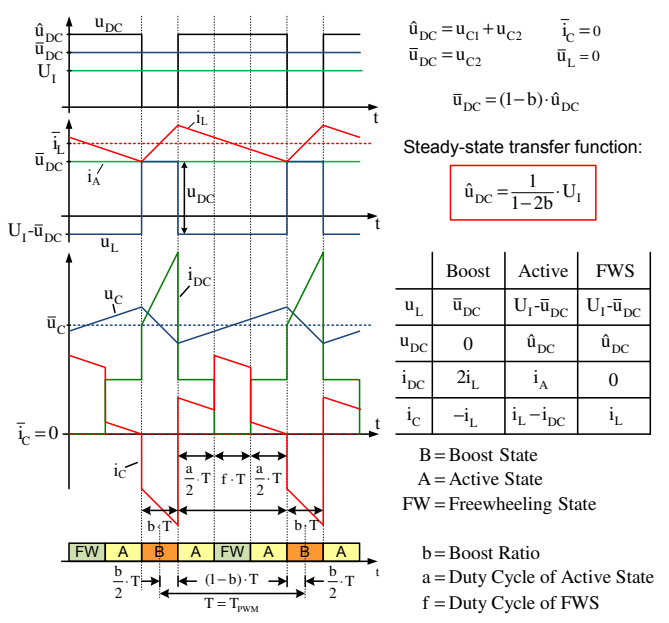

Figure 2. Steady-state boost operating mode of the QZSI

The only possibility to intervene in the circuit, is to alter the boost-cycle b, which is defined as the ratio of the time of the boost-state to one PWM-period:

$$
b=\frac{T_{\text {Boost }}}{T_{P W M}}
$$


Altering $\mathrm{b}$ changes the magnetic flux in the inductors and hence the amount of energy brought into the circuit. For the review on the possible switching states, the following assumptions are made:

- The components are ideal

- The inductances and the capacitances are each equal ( $L_{1}=L_{2}$ and $C_{1}=C_{2}$ )

- The inductances and the capacitances are designed sufficient, so that the short term average values of inductor currents and the capacitor voltages are approximately constant from one PWM-Period to the next.

- The supply voltage is constant

- The forward voltage drop of the diode is neglected

The inductances and capacitances must be large enough, so that the resonant frequency of the LC circuit is far lower than the switching frequency, to avoid oscillations. Then the time values of the inductor currents and capacitor voltages can be considered as being linear in any switching state. The influence of part variance $\left(L_{1} \neq L_{2}\right.$ and $C_{1} \neq C_{2}$ ) is neglected in this analysis. It can be shown that this has no effect on steady state condition, but leads to decaying oscillations in transient. In simulation, different values up to $20 \%$ led to decaying oscillations with amplitudes that are certainly within the range of a reasonable design. Those effects could be observed in the tested hardware and validated the simulation results.

In steady-state, the average voltages across the inductors and the average currents through the capacitors are zero, which leads to the following steady-state relations:

$$
\begin{aligned}
\bar{u}_{D C} & =U_{I}+\bar{u}_{C 1}=\bar{u}_{C 2} \\
\bar{i}_{L 1} & =\bar{i}_{L 2}=\bar{i}_{D}=\bar{i}_{D C}
\end{aligned}
$$

\section{A. Boost}

The boost-state is achieved by short-circuiting the DC bus through at least one leg of the three-phase bridge. The DC bus voltage then is zero and no power is transferred into the load. For the load this has the same effect as applying one of the two zero space vectors of conventional SVM. This makes it inevitable to consider the necessary boost time $T_{\text {Boost }}$ in the calculation of the load SVM.

$L_{2}$ and $C_{1}$ are connected to GND and the sum of the two capacitor voltages is applied to the diode as reverse voltage. Before the QZSI enters normal operating mode, the capacitor $C_{2}$ is pre-charged to the level of the input voltage. Hence, $u_{C 1}+u_{C 2}$ is far greater than zero and the diode blocks immediately in the first boost, applied to the circuit after precharging. During normal boost operating mode this sum even increases because of the boosted voltage and the diode blocks every time, the boost is applied. The equivalent circuit of the QZSI during boost is shown in fig. 3. With the assumption that the capacitor voltages are approximately equal from one period to the next, across both inductors the same voltage is applied (see also (2)) and the inductor currents are both increasing.

$$
\begin{aligned}
& u_{L 1}=U_{I}+u_{C 1} \approx U_{I}+\bar{u}_{C 1}=\bar{u}_{D C} \\
& u_{L 2}=u_{C 2} \approx \bar{u}_{C 2}=\bar{u}_{D C}
\end{aligned}
$$

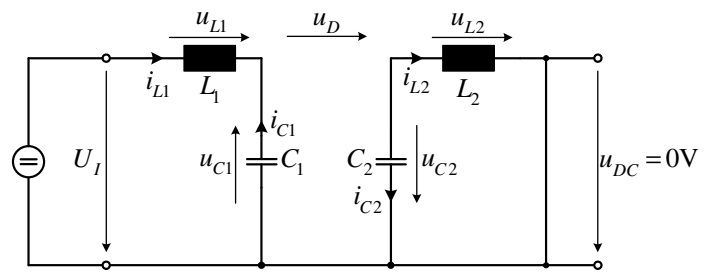

Figure 3. Equivalent circuit of the QZSI in boost state

With $L_{1}=L_{2}$ the inductor currents are equal. They flow as negative currents in the capacitors and discharge them partially.

$$
i_{L 1}=i_{L 2}=i_{L} \quad i_{C 1}=-i_{L 1} \quad i_{C 2}=-i_{L 2}
$$

With $C_{1}=C_{2}$ the voltage drop in both capacitors is equal too $\left(\Delta u_{C 1}=\Delta u_{C 2}\right)$. Since $C_{1}$ and $L_{2}$ are grounded via the same physical connection, the DC link current during boost equates to the doubled inductor current.

$$
i_{D C}=i_{L 1}+i_{L 2}=2 \cdot i_{L}
$$

\section{B. Boost with conducting diode}

During boost, the diode can only become conducting, if the sum of the two capacitor voltages becomes negative.

$$
u_{D}=-\left(u_{C 1}+u_{C 2}\right)>0 \quad \Rightarrow \quad-u_{C 1} \geq u_{C 2}
$$

This occurs, if the boost lasts longer than the discharging of the capacitors into the inductors or, in other words, longer than a quarter period of the LC series resonant circuit. During boost, the energy stored in the capacitors resonates into the two inductors. Since both capacitor voltages are decreasing equally (see II-A), starting with $u_{C 2}=U_{I}+\Delta u_{C}$ and $u_{C 1}=\Delta u_{C}$, the voltages applied to the inductors remain equal, resulting in equal inductor currents again. With the decreasing capacitor voltages, the potentials on the diode are changing; the anode potential rises with $-u_{C 1}(t)$, and the cathode potential decreases with $u_{C 2}(t)$. The condition for the conduction of the diode (7) is true, as soon as both capacitor voltages reach an absolute value of $\frac{1}{2} U_{I}$. With the diode conducting, the circuit can be drawn as shown in fig. 4. $C_{1}$ and $C_{2}$ are clamped onto each other by the diode, and the voltages remain constant

$$
u_{C 1}=u_{C 2}=u_{L 1}=u_{L 2}=\frac{1}{2} U_{I}
$$

The inductor currents are both continuously rising, only limited by the parasitic resistances. Hence this switching state must be avoided. Under normal operating conditions and precharging of $C_{2}$, this switching state doesn't occur (see II-A).

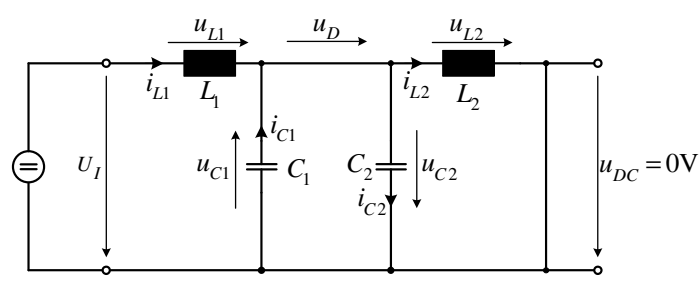

Figure 4. Equivalent circuit of the QZSI in boost-state with conducting diode 


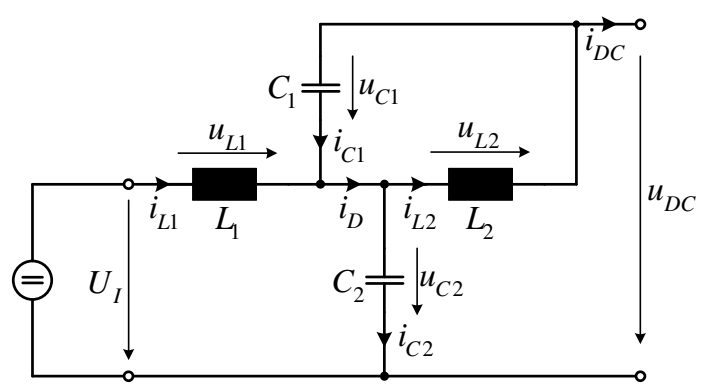

Figure 5. Equivalent circuit of the QZSI in active state

\section{Active State}

In active state, the shortcircuit is released and the load is connected to the DC link bus. The DC link potential, and with that the anode potential of the QZSI-diode, is not clamped anymore. Due to the now decreasing inductor currents that were impressed during the preceding boost-state, the DC link potential is rising, until the anode potential of the diode is equal to $u_{C 2}$ and the diode is conducting. Now the potentials are clamped again and the DC link voltage equals the sum of both capacitor voltages.

$$
u_{D C}=\hat{u}_{D C}=u_{C 1}+u_{C 2}
$$

The DC link then represents a capacitive voltage source for the load (fig. 5). The same voltage

$$
u_{L 1}=U_{I}-u_{C 2}=-u_{C 1}=u_{L 2}
$$

is applied to both inductors and the inductor currents are decreasing equally $\left(i_{L 1}=i_{L 2}=i_{L}\right)$. The diode current equals the sum of both inductor currents, reduced by the DC link current. The two capacitor currents are also equal again (see fig. 5).

$$
\begin{aligned}
i_{D} & =i_{L 1}+i_{C 1}=i_{L 1}+i_{L 2}-i_{D C}=2 i_{L}-i_{D C} \\
i_{C 1} & =i_{C 2}=i_{L}-i_{D C}
\end{aligned}
$$

The voltages across the capacitors equally depend on the difference between the inductor current and the DC link current. In steady-state operating mode the inductor current is greater than the DC link current and the capacitor voltages are rising again, after having been unloaded partially during boost. In transient, the current after a load step is initially provided through the capacitors, resulting in a voltage drop. The average inductor current is increased by the control loop, so that the DC link voltage reaches its specified value again.

\section{Freewheeling State (FWS)}

In freewheeling state no power is transferred into the load. This state is characterized by the absence of any connection between both DC link terminals, resulting in the DC link current becoming zero. The QZSI can be brought into this state by either turning off all switches of the three phase bridge or by connecting all load phases to either the positive or the negative DC link terminal (zero-space-vectors from conventional SVM). For the QZSI it doesn't matter which of those three actions lead to the freewheeling state - the DC link current is zero anyway. Since the DC bus is floating, the diode is conducting like in active state and fig. 5 with $i_{D C}=0$ is the resulting equivalent circuit. The capacitor currents equal the inductor currents

$$
i_{C 1}=i_{L 2} \quad \text { and } \quad i_{C 2}=i_{L 1}+i_{C 1}-i_{L 2}=i_{L 1}
$$

They can be determined by the applied voltages and like in all other switching states, the time values of inductor and capacitor currents and voltages are equal in this state too. The diode current equals the doubled inductor current.

$$
\begin{aligned}
u_{L 1} & =U_{I}-u_{C 2}=-u_{C 1}=u_{L 2} \\
i_{L 1} & =i_{L 2}=i_{L}=i_{C 1}=i_{C 2} \Rightarrow \Delta u_{C 1}=\Delta u_{C 2} \\
i_{D} & =2 i_{L}
\end{aligned}
$$

The equations show that the freewheeling state has the same effect on the QZSI like the active state with $i_{D C}=0$.

\section{E. Permanent Freewheeling State}

If the QZSI is driven in a permanent FWS, the energy stored in the inductors is being transferred completely into the capacitors. The time until the inductor current becomes zero, beginning with its nominal value, is

$$
\Delta t_{P F W S}=L \cdot \frac{\bar{i}_{L, N}}{u_{C 1, N}}
$$

The additional voltage on the capacitors is

$$
\Delta u_{C, P F W S}=\frac{1}{C} \cdot \frac{1}{2} \cdot \Delta i_{L} \cdot \Delta t_{P F W S}
$$

Because both capacitor currents are equal during FWS, this value is equal for both capacitors too. This voltage must be regarded in capacitor design. For the described QZSIprototype, the max. additional capacitor voltage would be $29,4 \mathrm{~V}$. Once the inductor current reaches zero, the LC series resonant circuit would make the inductor current become negative. However, this is impossible because of the diode. It blocks and the LC series resonant circuit is interrupted. The inductors remain currentless and the capacitors are charged with the controlled voltage plus the additional voltage. The DC link voltage drops to the level of $u_{C 2}$, as soon as the diode blocks. In contrary to a permanent boost, the permanent FWS is a secure switching state, into which the QZSI can be driven in any fault condition, for example by turning off all switches of the three phase bridge.

\section{F. Load-current caused boost (LCC)}

For the following explanation, a one-phase inductive load with $L_{A} \gg L_{Q Z S I}$ is assumed (see fig. 6). The FWS, as well as the boost act as freewheeling condition for the load. If the freewheeling current of the load is not yet zero at the end of a boost or a FWS, the freewheeling diode $\left(S_{L O}\right)$ still is conducting. The activation of the subsequent active state then provokes a shootthrough, since the upper switch and the lower diode are conducting at the same time, causing an unwanted boost-state and by that an unwanted voltage boost. If the freewheeling current $i_{F}$ can commutate instantly into the 


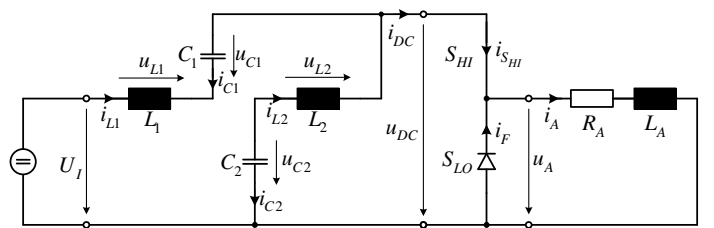

Figure 6. Equivalent Circuit of the QZSI in the LCC-boost state

upper bridge section, the duration of this load-current caused boost is close to zero and has no further effect. The condition for this instant commutation is that $i_{D C}$ must be at least as large as the output load current $i_{A}$.

$$
i_{A}<i_{D C, \text { Boost }}=2 i_{L}
$$

With the law of energy conservation, this can be used to determine the ratio between output and input voltage as condition for the instant commutation.

$$
\frac{\bar{u}_{A}}{U_{I}}=\frac{i_{I}}{i_{A}}>\frac{1}{2}
$$

This means that the average output voltage $\bar{u}_{A}$ must be at least half the input voltage. (20) can be rewritten for the duty cycle a, which is defined as the ratio of the time of the active state to the whole PWM-period:

$$
\begin{aligned}
& a=\frac{T_{\text {active }}}{T_{P W M}}=\frac{\bar{u}_{A}}{\hat{u}_{D C}} \Rightarrow \frac{\bar{u}_{A}}{U_{I}}=\frac{a \cdot \hat{u}_{D C}}{U_{I}}>\frac{1}{2} \\
& a>\frac{1}{2} \cdot \frac{U_{I}}{\hat{u}_{D C}}=\frac{1-2 b}{2}
\end{aligned}
$$

If this condition is not given, the LCC boost lasts, until the DC link current reaches the level of the freewheeling current. This is visible between $t_{1}$ and $t_{2}$ in fig. 7. At $t_{2}, i_{F}$ is zero and the freewheeling diode blocks. The QZSI changes to active state and the inductor currents are decreasing. This results in $i_{D C}$ becoming smaller than $i_{A}$, and the freewheeling diode becomes conducting again, resulting in another unwanted boost.

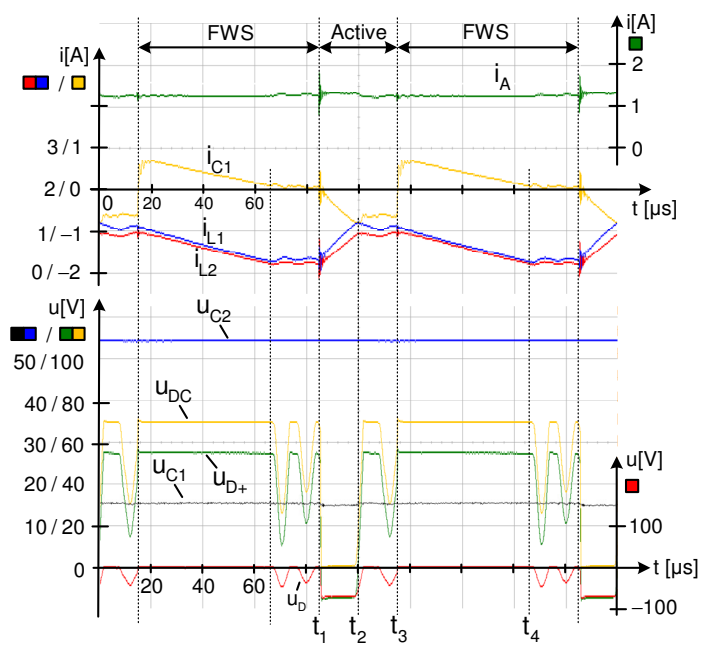

Figure 7. Oscillogramm of the load current caused boost at $U_{I}=40 \mathrm{~V}$ and $u_{C 2}=\bar{u}_{D C}=60 \mathrm{~V}$

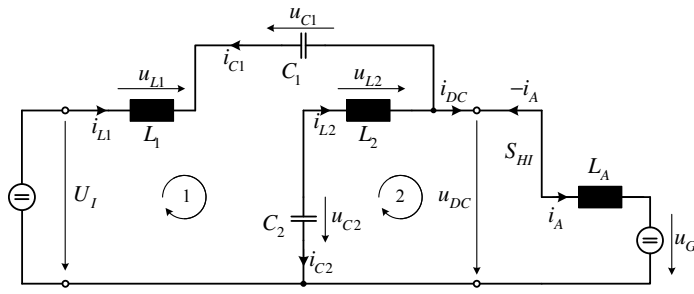

Figure 8. Equivalent circuit of the QZSI in feedback mode with open $S_{F B}$

At $t_{3}$, the output inverter is driven into FWS and the relations described in II-D are effective. Once the inductor current becomes zero $\left(t_{4}\right)$, the capacitor current is zero too. Since real components never are identical, there are oscillations between the passive components in this time interval (after $t_{4}$ ).

To be able to control the QZSI in the full voltage range, the LCC boost must be avoided. This can be achieved by turning on the feedback switch every time, the QZSI is driven in an active state. By doing that, the diode is bypassed and the DC bus is capacitive. Thus, the current to allow the instant commutation of the freewheeling current is provided. This method was tested with the described hardware and disabled the LCC boost effectively.

\section{G. Feedback mode}

In feedback mode, the load acts as current source. Due to the diode, the feedback current can't flow back into the input source. Initially, the feedback switch $S_{F B}$ (fig. 1) still is open, and the feedback current flows over $L_{1}-C_{1}$ and $L_{2}-C_{2}$. The inductor voltages are equal again (see fig. 8 and (2)).

$$
u_{L 1}=u_{L 2}=U_{I}+u_{C 1}-u_{D C}
$$

The feedback current is divided into two equal currents in each branch and the capacitor voltages are both rising equally. To prevent damage, the feedback switch must be closed if a certain maximum level of capacitor voltage is reached. Then the feedback current flows over $L_{2}-S_{F B}-L_{1}$ and feeds the input source. The energy stored in the capacitors starts to swing into the inductors, producing a resonant current that is superposed to the feedback current. By varying the duty cycle of the feedback switch, the voltage across the capacitors can be controlled in a way that the capacitor voltage that was controlled in boost mode, is reached again.

\section{DYNAMIC MODEL OF THE QZSI}

In chapter II was shown, that in the QZSI with $L_{1}=L_{2}$ and $C_{1}=C_{2}$, the two inductors are both supplied to identical voltages at any time in any switching state, leading to identical inductor currents as well. As result, the currents of the capacitors are equal in any switching state too. The voltages across the two capacitors differ only in the DC offset of the input voltage $U_{I}$, caused by the pre-charging of $C_{2}$.

$$
\begin{aligned}
u_{L 1}(t) & =u_{L 2}(t) & i_{L 1}(t) & =i_{L 2}(t) \\
\Delta u_{C 1}(t) & =\Delta u_{C 2}(t) & & i_{C 1}(t)=i_{C 2}(t)
\end{aligned}
$$


Because of that, the inductors and capacitors can be considered as two dependent parallel inductive and two dependent parallel capacitive energy storages.

With the assumptions made in chapter II, the time values of the capacitor voltages and the inductor currents can be considered as approximately constant from one PWM period to the next and approximately equal the corresponding short term average values over one PWM period:

$$
i_{L 1}=i_{L 2} \cong \bar{i}_{L} \quad u_{C 1} \cong \bar{u}_{C 1} \quad u_{C 2} \cong \bar{u}_{C 2}
$$

A. Inductor currents as a function of the boost cycle

As already mentioned, the only possibility to intervene in the circuit, is to alter the boost cycle b, which makes it essential to analyze the effect of a changing boost-cycle on the QZSI system dynamics. The short term average DC link voltage is (see fig. 2)

$$
\bar{u}_{D C}=(1-b) \cdot \hat{u}_{D C}
$$

In steady-state condition, this equals the voltage across $C_{2}$. The short term average voltage across $L_{2}$ is generally

$$
\bar{u}_{L 2}=\bar{u}_{C 2}-\bar{u}_{D C}=\bar{u}_{C 2}-(1-b) \cdot \hat{u}_{D C}
$$

If the boost-cycle is changed from an old value $b_{\text {old }}$ to a new value $b_{n e w}$, the difference of the voltages across the capacitors from one PWM period to the next can be neglected. Together with (2), the voltage across $C_{2}$ is

$$
\bar{u}_{C 2, \text { old }} \approx \bar{u}_{C 2, \text { new }} \approx \bar{u}_{C 2}=\bar{u}_{D C}
$$

The short term average value of the DC link voltage in contrary is changed immediately. With $b_{\text {new }}=b_{\text {old }}+\Delta b$, the short term average value of the voltage across $L_{2}$ is

$$
\begin{aligned}
\bar{u}_{L 2, \text { old }} & =\bar{u}_{C 2}-\bar{u}_{D C, \text { old }} \\
& =\left(1-b_{\text {old }}\right) \cdot \hat{u}_{D C}-\left(1-b_{\text {old }}\right) \cdot \hat{u}_{D C}=0 \\
\bar{u}_{L 2, \text { new }} & =\bar{u}_{C 2}-\bar{u}_{D C, \text { new }} \\
& =\left(1-b_{\text {old }}\right) \cdot \hat{u}_{D C}-\left(1-b_{\text {new }}\right) \cdot \hat{u}_{D C} \\
& =\left(-b_{\text {old }}+b_{\text {new }}\right) \cdot \hat{u}_{D C}=\Delta b \cdot \hat{u}_{D C} \\
\Delta \bar{u}_{L 2} & =\bar{u}_{L 2, \text { new }}-\bar{u}_{L 2, \text { old }}=\Delta b \cdot \hat{u}_{D C}
\end{aligned}
$$

This shows, that changing the boost-cycle $\mathrm{b}$ directly provokes a change of $\bar{u}_{L 2}$. The maximum DC link voltage acts as the actuator gain $V_{a c t}=\hat{u}_{D C}$. Since this voltage consists of the two capacitor voltages, it also can be considered as approximately constant over a few PWM periods.

The diode blocks only during boost, so the short term average voltage across the diode is then

$$
\bar{u}_{D}=b \cdot\left(-\hat{u}_{D C}\right)
$$

With the same assumptions concerning the capacitor voltages, the short term average voltage across $L_{1}$ is

$$
\bar{u}_{L 1}=U_{I}-\bar{u}_{C 2}-\bar{u}_{D}=U_{I}-\bar{u}_{C 2}+b \cdot \hat{u}_{D C}
$$

With $b_{\text {old }}$ the steady-state value of $\bar{u}_{L 1, \text { old }}$ is

$$
\begin{aligned}
\bar{u}_{L 1, \text { old }} & =U_{I}-\left(1-b_{\text {old }}\right) \cdot \hat{u}_{D C}+b_{\text {old }} \cdot \hat{u}_{D C} \\
& =U_{I}-\left(1-2 b_{\text {old }}\right) \cdot \hat{u}_{D C} \stackrel{!}{=} 0
\end{aligned}
$$

From (33), the steady state transfer function of the QZSI can directly be derived

$$
\frac{\hat{u}_{D C}}{U_{I}}=\frac{1}{(1-2 b)}
$$

The new short term average voltage across $L_{1}$, provoked by $b_{n e w}$ and the difference $\Delta \bar{u}_{L 1}$, is

$$
\begin{aligned}
\bar{u}_{L 1, \text { new }} & =U_{I}-\left(1-b_{\text {old }}\right) \cdot \hat{u}_{D C}+\left(b_{\text {old }}+\Delta b\right) \cdot \hat{u}_{D C} \\
& =\bar{u}_{L 1, \text { old }}+\Delta b \cdot \hat{u}_{D C} \\
\Delta \bar{u}_{L 1} & =\bar{u}_{L 1, \text { new }}-\bar{u}_{L 1, \text { old }}=\Delta b \cdot \hat{u}_{D C}
\end{aligned}
$$

The equations show, that for both inductors the same dynamic dependency between boost-cycle and the short term average voltage is effective. Altering the boost-cycle provokes for both inductors an identical change of the corresponding magnetic flux. By neglecting the series resistances, the inductors are modeled as integrators, which integrate the voltages to the corresponding inductor currents.

\section{B. Capacitor voltages as function of inductor currents}

The diode is only conducting in the active and in the freewheeling state. The diode current is

$$
i_{D}=\left\{\begin{array}{cl}
0 & \text { during boost } \\
i_{L 1}+i_{L 2}-i_{D C} & \text { during active state } \\
i_{L 1}+i_{L 2} & \text { during freewheeling state }
\end{array}\right.
$$

The diode current during freewheeling state differs from the one during active state only in the missing DC link current $\left(i_{D C}=0\right.$ in FWS $)$. The duration of the FWS is determined by the load control loop and is not controllable by the QZSIcontrol. Hence, $i_{D C}$ contributes as disturbance value. Together with the short term average value of $i_{D C}$, the short term average value of the diode current can be written as follows

$$
\begin{aligned}
& i_{D}=\left\{\begin{array}{cc}
0 & \text { during boost } \\
i_{L 1}+i_{L 2}-\bar{i}_{D C} & \text { rest of time }
\end{array}\right. \\
& \bar{i}_{D}=(1-b) \cdot\left(\bar{i}_{L 1}+\bar{i}_{L 2}-\bar{i}_{D C}\right)
\end{aligned}
$$

Given that the short term average values of the inductor currents are approximately constant from one PWM period to the next, the capacitor currents are (see fig. 1)

$$
\begin{aligned}
\bar{i}_{C 1}=\bar{i}_{D}-\bar{i}_{L 1} & =(1-b) \cdot\left(\bar{i}_{L 1}+\bar{i}_{L 2}-\bar{i}_{D C}\right)-\bar{i}_{L 1} \\
& =-b \cdot \bar{i}_{L 1}+(1-b) \cdot\left(\bar{i}_{L 2}-\bar{i}_{D C}\right) \\
\bar{i}_{C 2}=\bar{i}_{D}-\bar{i}_{L 2} & =(1-b) \cdot\left(\bar{i}_{L 1}+\bar{i}_{L 2}-\bar{i}_{D C}\right)-\bar{i}_{L 2} \\
& =-b \cdot \bar{i}_{L 2}+(1-b) \cdot\left(\bar{i}_{L 1}-\bar{i}_{D C}\right)
\end{aligned}
$$

Following these equations, the capacitor currents depend on the inductor currents and the DC link current, linked by the boost-cycle. The capacitor voltages are built through integration of the corresponding capacitor currents. The block diagram of the gained dynamic model of the QZSI is shown in fig. 9. It is also valid for components that have not the same values ( $L_{1} \neq L_{2}$ and $C_{1} \neq C_{2}$ ), as long as the assumption of an approximately constant inductor current and an approximately constant capacitor voltage from one PWM period to the next is valid. 


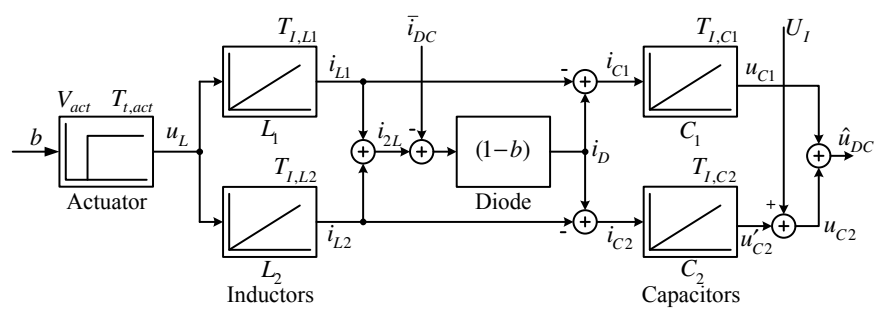

Figure 9. Dynamic average model of the QZSI

\section{Identical values in passive components}

If the passive components each have identical values ( $L_{1}=L_{2}$ and $C_{1}=C_{2}$ ), the model can be simplified significantly. For identical inductances, the inductor currents are equal $\left(i_{L 1}=i_{L 2}=i_{L}\right)$, and the sum $i_{2 L}$ of both can be written as

$$
i_{2 L}=i_{L 1}+i_{L 2}=2 i_{L}=\left(\frac{1}{s L_{1}}+\frac{1}{s L_{2}}\right) u_{L}=\frac{2}{s L} u_{L}
$$

The two parallel integrators can be considered as a single one with the integration constant of one inductor, and an adjacent proportional element with the gain $V_{P}=2$. With that, the diode and capacitor currents are

$$
\begin{aligned}
\bar{i}_{D} & =(1-b)\left(\bar{i}_{L 1}+\bar{i}_{L 2}-\bar{i}_{D C}\right)=(1-b)\left(\bar{i}_{2 L}-\bar{i}_{D C}\right) \\
\bar{i}_{C 1} & =\bar{i}_{D}-\bar{i}_{L 1}=\bar{i}_{D}-\bar{i}_{L 2}=\bar{i}_{C 2}=\bar{i}_{C}
\end{aligned}
$$

The equations of the capacitor currents can be rearranged, which leads to an expression that allows the separate examination of either command input or disturbance response.

$$
\begin{aligned}
\bar{i}_{C} & =\bar{i}_{C 1}=\bar{i}_{C 2}=\bar{i}_{D}-\bar{i}_{L} \\
& =(1-b) \cdot 2 \bar{i}_{L}-(1-b) \cdot \bar{i}_{D C}-\bar{i}_{L} \\
& =\left(\frac{1}{2}-b\right) \cdot 2 \bar{i}_{L}-(1-b) \cdot \bar{i}_{D C}
\end{aligned}
$$

The gained block diagram is shown in fig. 10. Since both capacitors are charged with the same current, they could also be rewritten as a single integrator, assumed that the capacitances are equal. Generally, for any values of the capacitances, the capacitor voltage is

$$
\bar{u}_{2 C}=\bar{u}_{C 1}+\bar{u}_{C 2}=\frac{1}{s} i_{C}\left(\frac{C_{1}+C_{2}}{C_{1} C_{2}}\right)
$$

However, this even further simplification is not pursued, because it shows that the control of $u_{C 2}$ has some advantages over the control of $\bar{u}_{2 C}$, mainly because $u_{C 2}$ is a DC value with only little ripple, can easily be measured and equals the average DC link voltage.

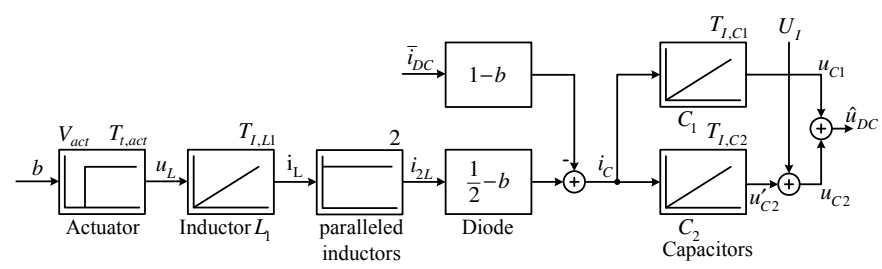

Figure 10. Dynamic average model of the QZSI with identical inductances and different capacitances

\section{EXPERIMENTAL RESULTS}

For the described model (fig. 10), a cascade control loop with inner current control loop $\left(i_{L}\right)$ and outer voltage control loop $\left(u_{C 2}=\bar{u}_{D C}\right)$ was developed and tested. As a brief proof of theory, the load step response of the complete QZSI control loop design with the described model as basis is shown in fig. 11. The oscillogram shows, that the proposed control method manages the load steps without any difficulty.

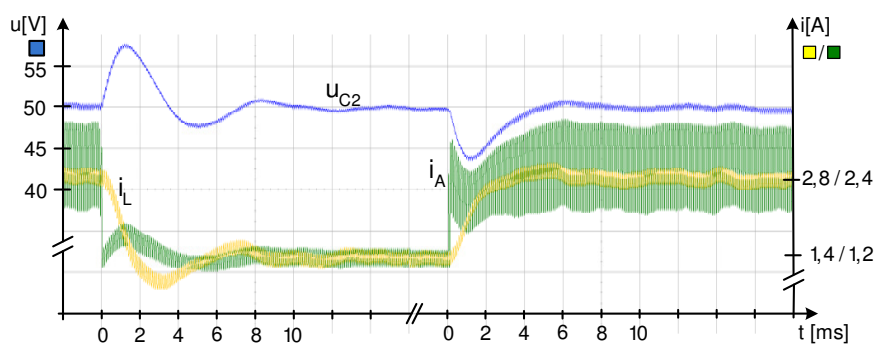

Figure 11. Step load change with resistive load. Nominal value of the DClink voltage $u_{C 2, w}=50 \mathrm{~V}$. Left: load rejection. Right: load surge.

\section{CONCLUSION}

The possible switching states that can occur in the QZSI were analyzed and described in order to study the dynamic behaviour of the QZSI. Based on this analysis, the short term average values over one PWM-period were calculated and a dynamic average model of the QZSI was developed. The described model is rather descriptive and has the advantage that the load influence acts only as disturbance input and is not directly linked with the model structure itself. This gives the freedom to design the control loop for the QZSI independently from the load. The model is valid under the assumption that the inductor currents and the capacitor voltages are approximately constant from one PWM-period to the next. This is normally the case, since the PWM switching frequency is significantly larger than the QZSI L and $\mathrm{C}$ time constants. For similar inductances and capacitances $\left(L_{1}=L_{2}\right.$ and $\left.C_{1}=C_{2}\right)$, a significantly simplified model could be derived. For this simplified model, a cascade control strategy was developed and successfully tested with a QZSI-prototype in hardware, which verifies the theory completely.

\section{REFERENCES}

[1] F. P. Joel Anderson, "Four quasi-z-source inverters." Michigan State University Department of Electrical and Computer Engineering, 2008

[2] M. v. Z. Lothar Sack, "Z-source-topologie als alternative zu herkömmlichen antriebsumrichtern," Präsentation, Kolloqium des Elektrotechnischen Instituts, 2009.

[3] F. Z. Peng, "Z-source inverter," IEEE Transactions on Industry Applications, Vol 39, No. 2, March/April 2003.

[4] R. M. Slobodan Cuk, "Modeling, analysis and design of switching converter," 1978.

[5] L. X. Jingbo Liu, Jiangang Hu, "Dynamic modeling and analysis of z source converter - derivation of ac small signal model and design-oriented analysis," IEEE, 2007.

[6] F. P. Miaosen Shen, Qingsong Tang, "Modeling and controller design of the z-source inverter with inductive load," IEEE, 2007.

[7] M. U. D.M. Vilathgamuwa, P.C. Loh, "Transient modelling and control of z-source current type inverter," IEEE, 2007. 